\title{
Resonance Analysis for Tilted Support Spring Coupled Nonlinear Packaging System Applying Variational Iteration Method
}

\author{
An-Jun Chen ${ }^{1,2}$ \\ ${ }^{1}$ Department of Packaging Engineering, Jiangnan University, Wuxi 214122, China \\ ${ }^{2}$ Key Laboratory of Food Packaging Techniques \& Safety of China National Packaging Corporation, Wuxi 214122, China
}

Correspondence should be addressed to An-Jun Chen; caj62@163.com

Received 15 January 2013; Accepted 24 April 2013

Academic Editor: Vanee Chonhenchob

Copyright (C) 2013 An-Jun Chen. This is an open access article distributed under the Creative Commons Attribution License, which permits unrestricted use, distribution, and reproduction in any medium, provided the original work is properly cited.

The coupled nonlinear dynamical equations were developed for a tilted spring packaging system with critical components. The approximate solution and resonance conditions of system were obtained applying a variational iteration method. The resonance conditions, which should be avoided in the packaging design, can be easily obtained by VIM.

\section{Introduction}

Damage of products frequently occurs at the circulating process, the vibration and shock are main dynamical reasons, it is very important to investigate the dynamical characteristics of packaging system for packaging design. Newton's damage boundary concept [1] and succeeding modified damage evaluation approaches [2-4] were widely utilized in packaging design. The cushioning pad was treated as a simple linear or nonlinear spring, and packaging system was considered to be a single degree of freedom system [5]. However, the real packaging system, like the tilted spring system which was demonstrated to be a better candidate for products protection than linear system [6-8], includes product and many critical components, which is multidegree-of-freedom system and nonlinear by nature. And it remains a problem to obtain the resonance condition for nonlinear packaging system, especially for multi-degreeof-freedom nonlinear cushioning packaging system. Most recently, various analytical approaches for solving nonlinear differential equations were widely applied in the analysis of engineering practical problems, such as EBM [9], PEM [10], and VIM [11, 12]. The variational iteration method does not need the assumption of linearization or weak nonlinearity and depends totally on Lagrange multiplier theory, which means that this method will only fail if the Lagrange multiplier for any partial differential equation/ordinary differential equation (PDE/ODE) or coupled PDE/ODE does not exist. Fortunately, the Lagrange multiplier for nonlinear equations can be simply obtained in most cases. In this paper, the variational iteration method was suggested to obtain the innerresonance condition for a strong nonlinear packaging system.

\section{Modeling and Equations}

The model of tilted spring system with critical components can be modeled as shown in Figure 1. The packaged product is supported by four springs which own the same stiffness $k_{2}$ and length $l_{0}, \varphi_{0}$ is the angle of primary support position, $m_{1}$ and $m_{2}$ denote the mass of the critical component and the main part of the product, and $k_{1}$ is the coupling stiffness of the critical component.

The dropping shock approximate dynamic equations of system can be expressed as

$$
\begin{gathered}
m_{1} \frac{\mathrm{d}^{2} x_{1}}{\mathrm{~d} t^{2}}-k_{1}\left(x_{2}-x_{1}\right)=0, \\
m_{2} \frac{\mathrm{d}^{2} x_{2}}{\mathrm{~d} t^{2}}+4 k_{2}\left(a_{0} x_{2}+\frac{b_{0}}{l_{0}} x_{2}^{2}+\frac{c_{0}}{l_{2}^{2}} x_{2}^{3}\right)+k_{1}\left(x_{2}-x_{1}\right)=0,
\end{gathered}
$$




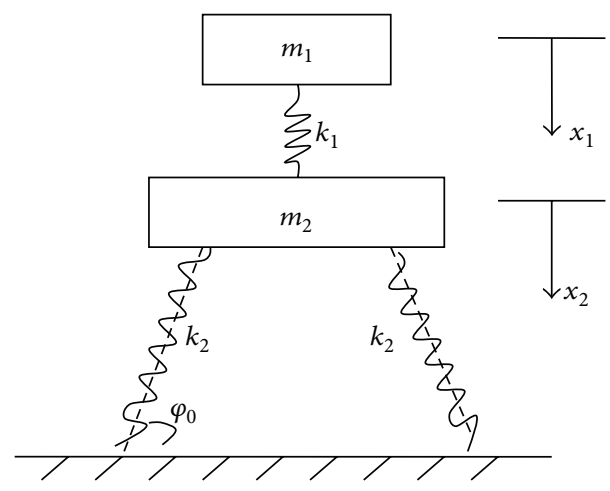

Figure 1: The model of tilted spring system.

with the initial conditions

$$
\begin{array}{ll}
x_{1}(0)=0, & \frac{\mathrm{d} x_{1}(0)}{\mathrm{d} t}=\sqrt{2 g h}, \\
x_{2}(0)=0, & \frac{\mathrm{d} x_{2}(0)}{\mathrm{d} t}=\sqrt{2 g h},
\end{array}
$$

where $a_{0}=\sin ^{2} \varphi_{0}, b_{0}=-(3 / 2) \sin \varphi_{0} \cos ^{2} \varphi_{0}, c_{0}=(1 / 2)(1-$ $\left.6 \sin ^{2} \varphi_{0}+5 \sin ^{4} \varphi_{0}\right), h$ is the dropping height, and $g$ is the gravity acceleration. Equation (1) can be rewritten in the following forms:

$$
\begin{gathered}
\frac{\mathrm{d}^{2} y_{1}}{\mathrm{~d} \tau^{2}}+\omega_{y 1}^{2} y_{1}-\omega_{y 1}^{2} y_{2}=0 \\
\frac{\mathrm{d}^{2} y_{2}}{\mathrm{~d} \tau^{2}}+\omega_{y 2}^{2} y_{2}+b_{0} y_{2}^{2}+c_{0} y_{2}^{3}+\left(a_{0}-\omega_{y 2}^{2}\right) y_{1}=0
\end{gathered}
$$

where

$$
\begin{gathered}
y_{1}=\frac{x_{1}}{l_{0}}, \quad y_{2}=\frac{x_{2}}{l_{0}}, \\
\omega_{y 1}=\lambda_{1}, \quad \omega_{y 2}=\sqrt{a_{0}+\lambda_{1}^{2} \lambda_{2}} .
\end{gathered}
$$

Here $y_{1}$ and $y_{2}$ are the dimensionless displacement parameters, $\omega_{1}=\sqrt{k_{1} / m_{1}}$ and $\omega_{2}=\sqrt{4 k_{2} / m_{2}}$ are the frequency parameters, $T=1 / \omega_{2}$ is the periodic parameter, $\tau=t / T$ is the dimensionless time parameter, $\lambda_{1}=\omega_{1} / \omega_{2}$ is the frequency ratio, and $\lambda_{2}=m_{1} / m_{2}$ is the mass ratio of system. The nondimensional form of the initial conditions can be written as

$$
\begin{array}{ll}
y_{1}(0)=0, & \frac{\mathrm{d} y_{1}(0)}{\mathrm{d} \tau}=\frac{T}{l_{0}} \sqrt{2 g h}, \\
y_{2}(0)=0, & \frac{\mathrm{d} y_{2}(0)}{\mathrm{d} \tau}=\frac{T}{l_{0}} \sqrt{2 g h} .
\end{array}
$$

\section{Variational Iteration Method}

Being different from the other nonlinear analytical methods, variational iteration method $[11,12]$ does not depend on small parameters, such that it can find wide application in nonlinear problem without linearization or small perturbations. Using the variational iteration method, we can construct the following iteration formula for (4):

$$
\begin{aligned}
y_{2 n+1}=y_{2 n}+\frac{1}{\omega_{y 2}} \int_{0}^{\tau} \sin [ & \left.\omega_{y 2}(s-\tau)\right] \\
\times & \frac{\mathrm{d}^{2} y_{2 n}}{\mathrm{~d} s^{2}}+\omega_{y 2}^{2} y_{2 n}+b_{0} y_{2 n}^{2} \\
& \left.+c_{0} y_{2 n}^{3}+\left(a_{0}-\omega_{y 2}^{2}\right) y_{1 n}\right) \mathrm{d} s .
\end{aligned}
$$

We choose the initial solution as

$$
\begin{aligned}
& y_{10}=A_{1} \sin \left(\Omega_{1} \tau\right), \\
& y_{20}=A_{2} \sin \left(\Omega_{2} \tau\right),
\end{aligned}
$$

where

$$
A_{1}=\frac{T}{l_{0} \Omega_{1}} \sqrt{2 g h}, \quad A_{2}=\frac{T}{l_{0} \Omega_{2}} \sqrt{2 g h} .
$$

By the iteration formula (8), we have the following firstorder approximation solution:

$$
\begin{aligned}
y_{21}= & \left(\frac{A_{2} \Omega_{2}}{\omega_{y 2}}+\frac{3 c_{0} A_{2}^{3} \Omega_{2}}{4 \omega_{y 2}\left(\omega_{y 2}^{2}-\Omega_{2}^{2}\right)}-\frac{3 c_{0} A_{2}^{3} \Omega_{2}}{4 \omega_{y 2}\left(\omega_{y 2}^{2}-9 \Omega_{2}^{2}\right)}\right. \\
& \left.+\frac{A_{1} \Omega_{1}\left(a_{0}-\omega_{y 2}^{2}\right)}{\omega_{y 2}\left(\omega_{y 2}^{2}-\Omega_{1}^{2}\right)}\right) \sin \left(\omega_{y 2} \tau\right) \\
& +\left(\frac{b_{0} A_{2}^{2}}{2 \omega_{y 2}^{2}}-\frac{b_{0} A_{2}^{2}}{\omega_{y 2}^{2}-4 \Omega_{2}^{2}}\right) \cos \left(\omega_{y 2} \tau\right) \\
& -\frac{A_{1}\left(a_{0}-\omega_{y 2}^{2}\right)}{\omega_{y 2}^{2}-\Omega_{1}^{2}} \sin \left(\Omega_{1} \tau\right) \\
& -\frac{3 c_{0} A_{2}^{3}}{4\left(\omega_{y 2}^{2}-\Omega_{2}^{2}\right)} \sin \left(\Omega_{2} \tau\right) \\
& +\frac{c_{0} A_{2}^{3}}{4\left(\omega_{y 2}^{2}-9 \Omega_{2}^{2}\right)} \sin \left(3 \Omega_{2} \tau\right) \\
+ & \frac{b_{0} A_{2}^{2}}{\omega_{y 2}^{2}-4 \Omega_{2}^{2}} \cos \left(2 \Omega_{2} \tau\right) \\
& \frac{b_{y 2}^{2}}{2}
\end{aligned}
$$


Substituting the first-order approximation solution of (11) into (3), the stationary process solution of (3) can be written as

$$
\begin{aligned}
y_{11}= & \frac{C_{1} \omega_{y 1}^{2}}{\omega_{y 1}^{2}-\omega_{y 2}^{2}} \sin \left(\omega_{y 2} \tau\right)+\frac{C_{2} \omega_{y 1}^{2}}{\omega_{y 1}^{2}-\omega_{y 2}^{2}} \cos \left(\omega_{y 2} \tau\right) \\
& +\frac{C_{3} \omega_{y 1}^{2}}{\omega_{y 1}^{2}-\Omega_{1}^{2}} \sin \left(\Omega_{1} \tau\right)+\frac{C_{4} \omega_{y 1}^{2}}{\omega_{y 1}^{2}-\Omega_{2}^{2}} \sin \left(\Omega_{2} \tau\right) \\
& +\frac{C_{5} \omega_{y 1}^{2}}{\omega_{y 1}^{2}-9 \Omega_{2}^{2}} \sin \left(3 \Omega_{2} \tau\right)+\frac{C_{6} \omega_{y 1}^{2}}{\omega_{y 1}^{2}-4 \Omega_{2}^{2}} \cos \left(2 \Omega_{2} \tau\right),
\end{aligned}
$$

where

$$
\begin{aligned}
C_{1}= & \frac{A_{2} \Omega_{2}}{\omega_{y 2}}+\frac{3 c_{0} A_{2}^{3} \Omega_{2}}{4 \omega_{y 2}\left(\omega_{y 2}^{2}-\Omega_{2}^{2}\right)} \\
& \quad-\frac{3 c_{0} A_{2}^{3} \Omega_{2}}{4 \omega_{y 2}\left(\omega_{y 2}^{2}-9 \Omega_{2}^{2}\right)}+\frac{A_{1} \Omega_{1}\left(a_{0}-\omega_{y 2}^{2}\right)}{\omega_{y 2}\left(\omega_{y 2}^{2}-\Omega_{1}^{2}\right)}, \\
C_{2}= & \frac{b_{0} A_{2}^{2}}{2 \omega_{y 2}^{2}-\frac{b_{0} A_{2}^{2}}{\omega_{y 2}^{2}-4 \Omega_{2}^{2}}} \\
C_{3}= & -\frac{A_{1}\left(a_{0}-\omega_{y 2}^{2}\right)}{\omega_{y 2}^{2}-\Omega_{1}^{2}} \\
C_{4}= & -\frac{3 c_{0} A_{2}^{3}}{4\left(\omega_{y 2}^{2}-\Omega_{2}^{2}\right)}, \\
C_{6}= & \frac{c_{0} A_{2}^{3}}{4\left(\omega_{y 2}^{2}-9 \Omega_{2}^{2}\right)} \\
\omega_{y 2}^{2}-4 \Omega_{2}^{2} &
\end{aligned}
$$

Computation illustrates that for the tilted spring system with critical components, the first-order approximation solution is enough. As shown in Figure 2, the nondimensional dropping shock response displacement $\left(y_{11}\right)$ of the critical components is calculated and compared with the numerical integration solutions by using a built-in ODE45 solver in MATLAB, showing good agreement. The results are obtained for the following amounts: $\lambda_{1}=10, \lambda_{2}=0.01$, and $\varphi_{0}=80^{\circ}$, and the initial conditions are $y_{1}(0)=y_{2}(0)=0, \mathrm{~d} y_{1}(0) / \mathrm{d} \tau=$ $\mathrm{d} y_{2}(0) / \mathrm{d} \tau=0.3$.

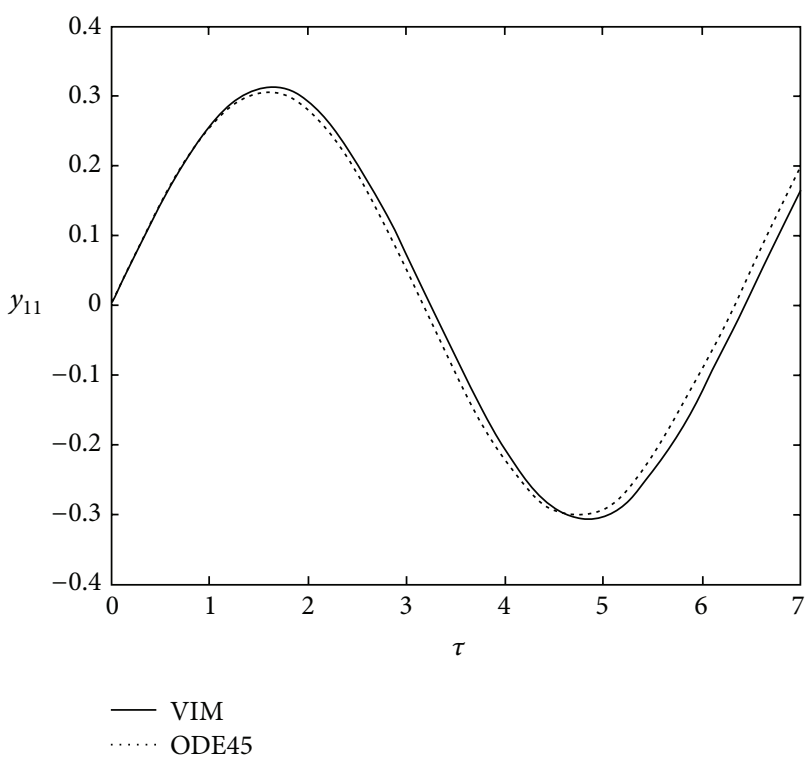

FIGURE 2: The comparison between the VIM solutions with ordinary differential equation solver in MATLAB.

\section{Resonance}

Through (11) and (12), the resonance conditions can be simplified as

$$
\begin{gathered}
\Omega_{1}=\omega_{y 2}, \\
\Omega_{2}=\omega_{y 2}, \\
\Omega_{2}=\frac{1}{2} \omega_{y 2}, \\
\Omega_{2}=\frac{1}{3} \omega_{y 2}, \\
\Omega_{1}=\omega_{y 1}, \\
\Omega_{2}=\omega_{y 1}, \\
\Omega_{2}=\frac{1}{2} \omega_{y 1}, \\
\Omega_{2}=\frac{1}{3} \omega_{y 1}, \\
\omega_{y 2}=\omega_{y 1} .
\end{gathered}
$$

These conditions should be avoided during the cushioning packaging design procedure.

\section{Conclusion}

Packaged products can be potentially dropped in the transportation, during which the packaged products may be seriously damaged by the inner-resonance, especially for those large and fragile products. Therefore, it is essential to obtain the inner resonance conditions for dropped packaging system. In this paper, the nonlinear dynamical equations were 
established for the tilted support spring system with critical components, and the resonance conditions of system are discussed by using the variational iteration method. The firstorder approximation solution was obtained and compared with the numerical simulation results using ODE45 solver in MATLAB, showing good agreement. The resonance conditions, which should be avoided in the packaging design, can be easily obtained by VIM.

\section{References}

[1] R. E. Newton, Fragility Assessment Theory and Practice, Monterey Research Laboratory, Monterey, Calif, USA, 1968.

[2] G. J. Burgess, "Products fragility and damage boundary theory," Packaging Technology and Science, vol. 15, no. 10, pp. 5-10, 1988.

[3] Z. Wang, C. Wu, and D. Xi, "Damage boundary of a packaging system under rectangular pulse excitation," Packaging Technology and Science, vol. 11, no. 4, pp. 189-202, 1998.

[4] J. Wang, F. Duan, J. Jiang et al., "Dropping damage evaluation for a hyperbolic tangent cushioning system with a critical component," Journal of Vibration and Control, vol. 18, no. 10, pp. 1417-1421, 2012.

[5] J. Wang, Z. W. Wang, L. X. Lu, Y. Zhu, and Y. G. Wang, "Three-dimensional shock spectrum of critical component for nonlinear packaging system," Shock and Vibration, vol. 18, no. 3 , pp. 437-445, 2011.

[6] X. Wu, Y.X. Luo, and Y. Wu, "Study on vertical nonlinear natural vibration of shock absorber system with tilted support spring," Journal of Vibration and Shock, vol. 27, no. 8, pp. 85-87, 2008.

[7] X. Wu, Y. X. Luo, and L. J. Yang, “The resonance property of shock absorber system with tilted spring under foundation displacement excitation," Journal of Beijing University of Technology, vol. 36, no. 12, pp. 1637-1641, 2010.

[8] A. J. Chen, "Shock characteristics of a tilted support spring system under action of a rectangular pulse," Journal of Vibration and Shock, vol. 29, no. 10, pp. 225-227, 2010.

[9] I. Mehdipour, D. D. Ganji, and M. Mozaffari, "Application of the energy balance method to nonlinear vibrating equations," Current Applied Physics, vol. 10, no. 1, pp. 104-112, 2010.

[10] A. Kimiaeifar, A. R. Saidi, A. R. Sohouli, and D. D. Ganji, "Analysis of modified Van der Pol's oscillator using He's parameterexpanding methods," Current Applied Physics, vol. 10, no. 1, pp. 279-283, 2010.

[11] J. H. He, "Variational iteration method-a kind of non-linear analytical technique: some examples," International Journal of Non-Linear Mechanics, vol. 34, no. 4, pp. 699-708, 1999.

[12] J. H. He, "Variational iteration method-some recent results and new interpretations," Journal of Computational and Applied Mathematics, vol. 207, no. 1, pp. 3-17, 2007. 


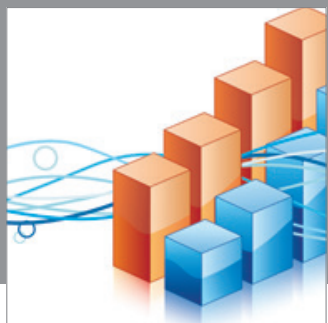

Advances in

Operations Research

mansans

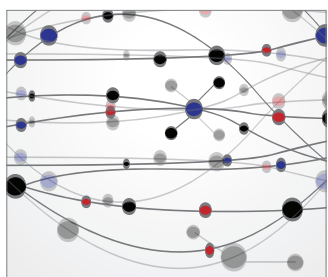

The Scientific World Journal
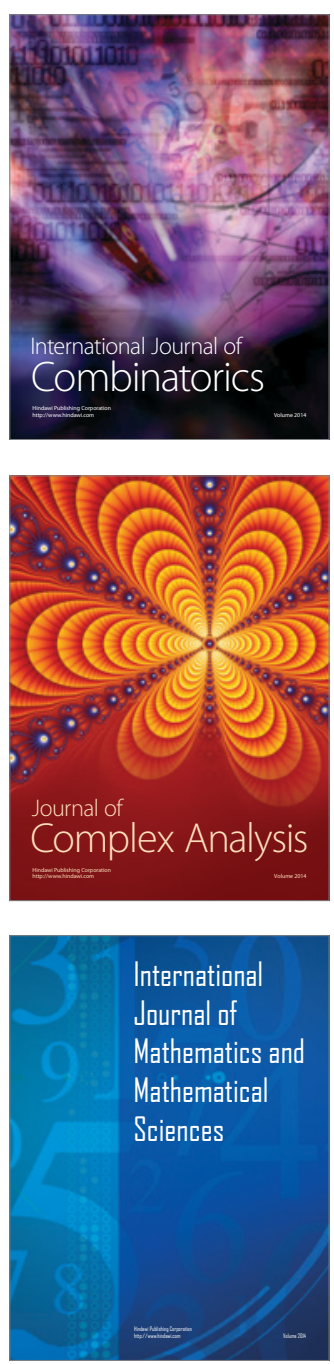
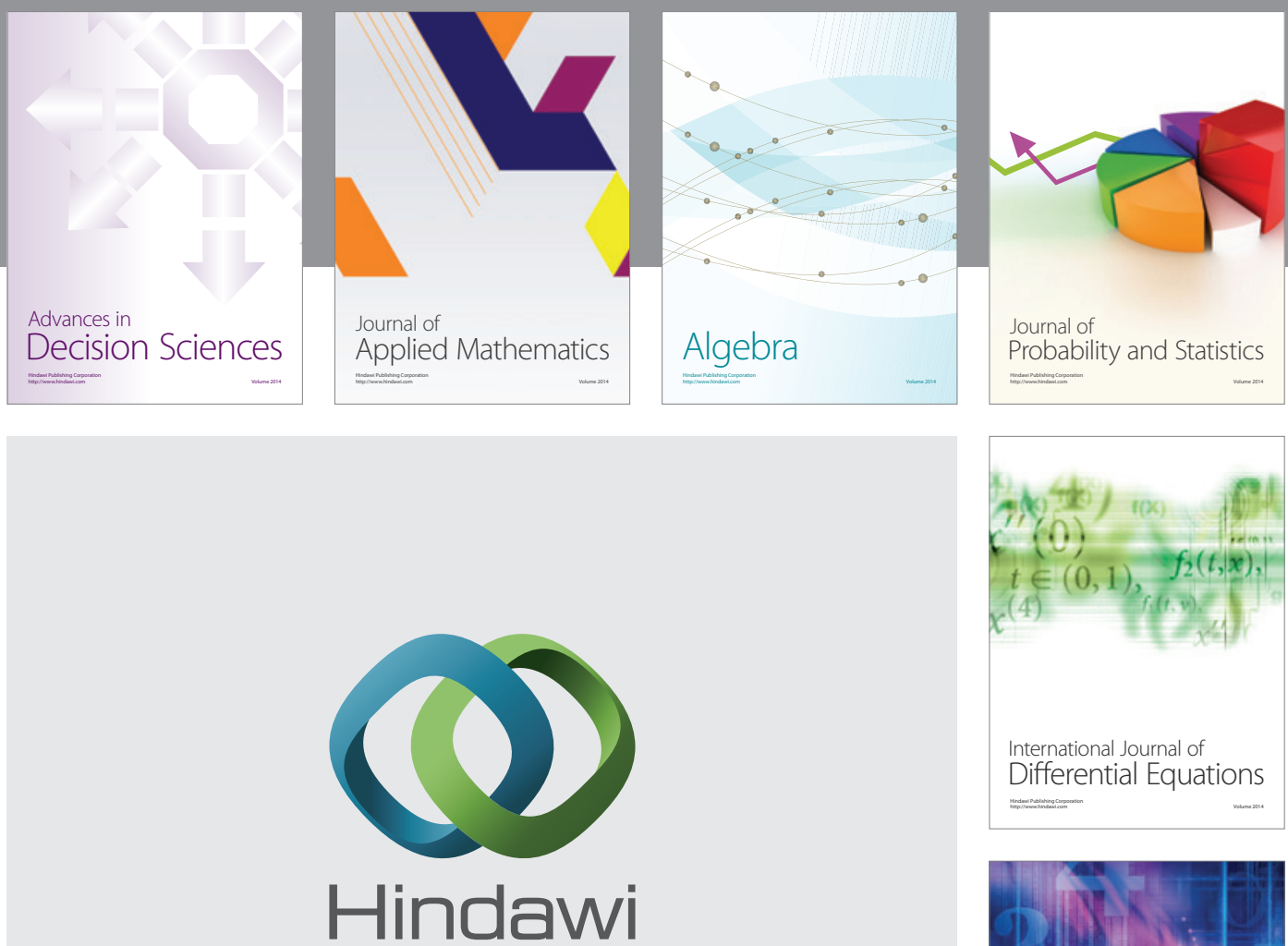

Submit your manuscripts at http://www.hindawi.com
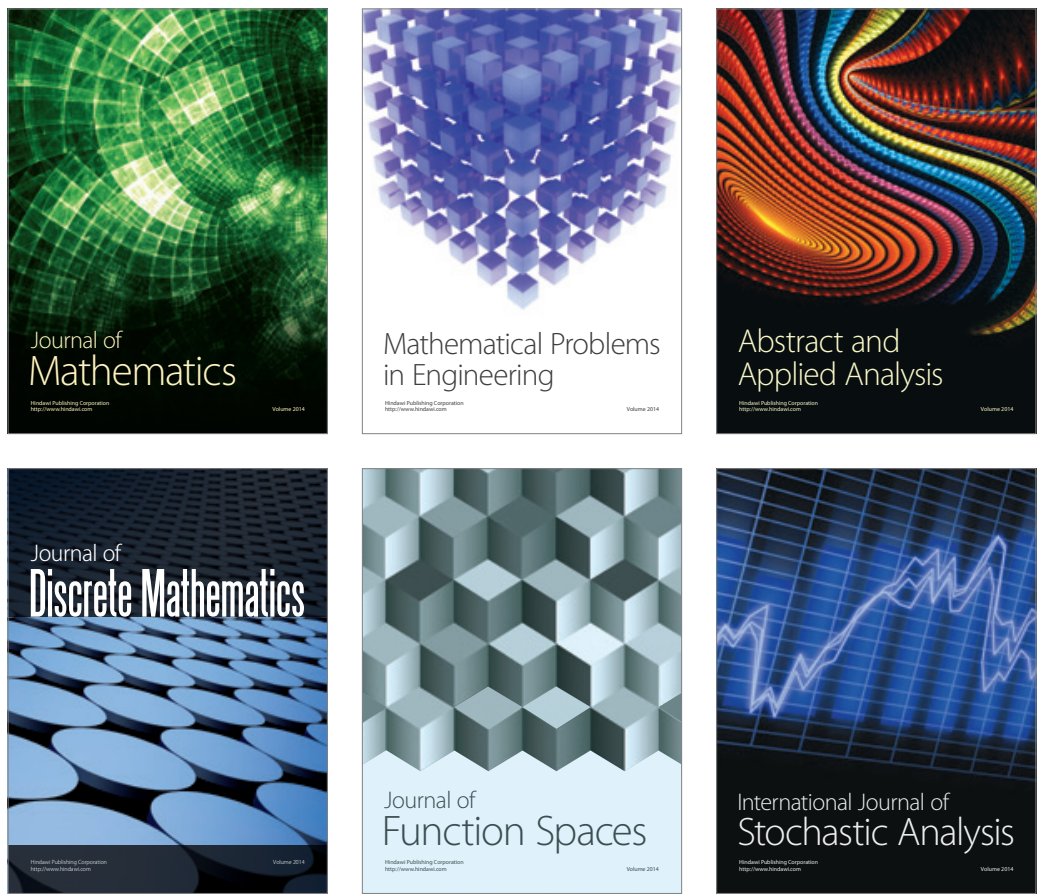

Journal of

Function Spaces

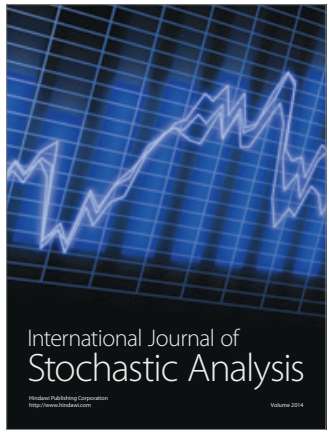

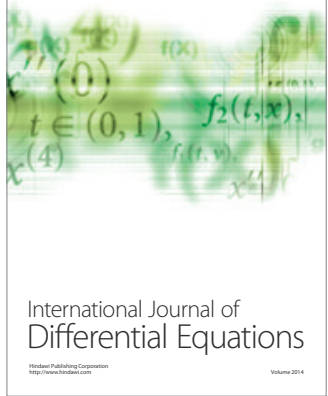
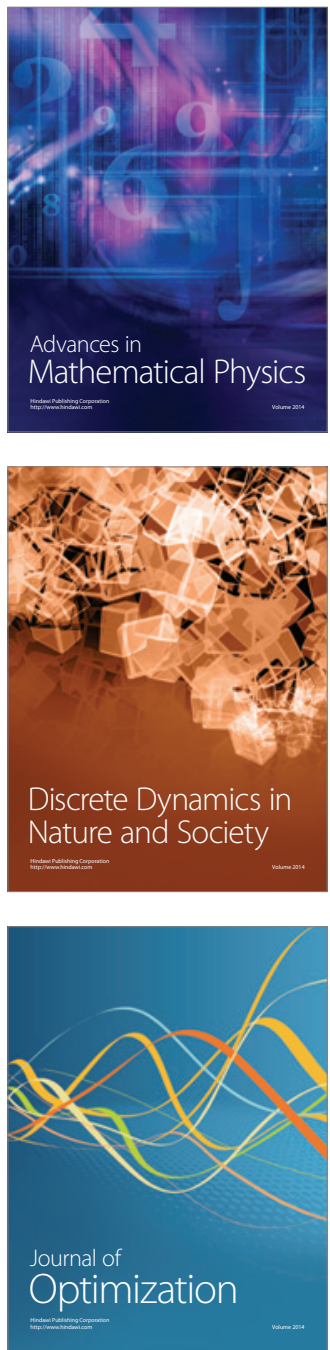